\title{
NOMA and Interference Limited Satellite Scenarios
}

\author{
Màrius Caus ${ }^{\star}$, Miguel Ángel Vázquez ${ }^{\star}$, Ana Pérez-Neira ${ }^{\star \dagger}$ \\ * Centre Tecnològic de Telecomunicacions de Catalunya (CTTC) \\ $\dagger$ Dept. of Signal Theory and Communications Universitat Politècnica de Catalunya \\ Email:\{marius.caus, mavazquez, aperez\}@cttc.cat
}

\begin{abstract}
This paper deals with the problem of nonorthogonal multiple access (NOMA) in multibeam satellite systems, where the signals are jointly precoded. It is considered that the number of frames that are simultaneously transmitted is higher than the number of feeds, reducing the precoding interference mitigation capabilities as the system becomes overloaded. In order to solve this problem, we assume that the satellite user terminals are able to perform multi-user detection to mitigate the interference. In the current NOMA approach, it is assumed a successive interference cancellation (SIC) receiver. To increase the spectral efficiency, this paper investigates NOMA with simultaneous non-unique detection (SND). Compared to the case where user terminals perform single user detection (SUD), conventional scheduling heuristic rules do not longer apply in this scenario. Therefore, different scheduling algorithms are proposed considering both SIC and SND strategies. As the numerical evaluations show, SND yields larger average data rates than the SIC receiver. Concerning the scheduling, the best strategy is to pair users with highly correlated channels and the lowest channel gain difference. It is also shown that the sum-rate can be increased in overloaded satellite systems with respect to satellite scenarios, where the number of transmitted frames and feeds is the same.
\end{abstract}

\section{INTRODUCTION}

The role of satellite communication systems is becoming more and more prominent, as the demand for higher data rates and ubiquity is increasing. Moreover, satellite systems are emerging as an attractive solution to offer connectivity to remote regions, where terrestrial network infrastructures are not deployed. Therefore, if $100 \%$ coverage is identified as a strategic requirement for coming soon $5 \mathrm{G}$ mobile networks, then it is crucial that the future architecture supports the integration of satellite and terrestrial networks. In order to keep the peace with user demands for broadband services, multibeam technology should be employed to reuse the frequency and, thus, increase the spectral efficiency.

To avoid inter-beam interference, it is customary to adopt a four-color beam pattern, so that adjacent beams operate on different frequencies. In this scenario, interference that comes from other beams is received with a magnitude significantly lower than that of the signal of interest and, thus, can be treated as noise. The spectral efficiency can be increased by considering a two-color beam pattern. Adopting more aggressive frequency reuse schemes, the signal bandwidth is increased, although the interference can be received with

This work has received funding from the European Unions Horizon2020 research and innovation programme under grant agreement No 645047(SANSA); the Spanish Ministry of Economy and Competitiveness (Ministeriode Economia y Competitividad) under project TEC2014-59255- C3-1-R (ELISA); and from the Catalan Government (2014SGR1567 and 2014SGR1551) the same level as the useful signal. Through cooperative transmission schemes and decentralized multi-user detection (MUD) techniques [1]-[3], the negative impact of interference can be mitigated to a high extent. To unleash the full potential of multibeam satellite systems and boost the capacity, all the beams have to share time and frequency resources, resulting in a one-color beam pattern. Clearly, this scenario is limited by the interference, which can only be effectively mitigated by relying on precoding techniques at the gateway [4]. Since the complexity of MUD techniques exponentially increases with the number of signals to be detected, it becomes evident that for complexity reasons the receiver cannot deal with all the interfering signals in full frequency reuse schemes.

In the light of the above discussion, it can be resolved that the most common approach to combat the detrimental effects of inter-beam interference, consists in either relying on precoding or MUD. Recently in [5], the authors have proposed to combine both techniques to increase the achievable rates. One of the most important conclusions that can be drawn from [5] is that the use of high performance receivers, which are capable of performing MUD, allow to overload the system. In other words, it is possible to simultaneously serve a number of users that is higher than the number of feeds. In this case, the receiver is able to cope with the strongest interfering signal that cannot be completely removed by precoding techniques.

The work presented in this paper focuses on studying the forward link of a multibeam satellite system that is overloaded. From this point onwards, we will refer to overloaded systems to indicate that two users located in the same spot-beam area are scheduled in the same time slots and share the available bandwidth. In each beam, the system model bears resemblance with the downlink of a cellular system that employs nonorthogonal multiple access (NOMA) [6].

NOMA, also known as multi-user superposition transmission (MUST), has attracted considerable attention, because superposition coding combined with the use of successive interference cancellation (SIC) or single user detection (SUD) at the receive side, keeps the complexity at a reasonable level. This paper proposes to enhance this scheme and apply NOMA to multibeam satellite transmission with the decoding strategies described in [7]. Leveraging the joint precoding and MUD techniques described in [5], the main contribution of this paper is the design of low-complexity scheduling algorithms for overloaded satellite systems. It is important to remark that the solutions devised for terrestrial communications, e.g. [8], cannot be directly applied to satellite systems, because the complexity scales with the number of beams. Therefore, simpler heuristic algorithms, which avoid exhaustive search, 
are proposed with the aim of maximizing the sum-rate and the fairness. Numerical results in closed-to-real overloaded satellite systems, reveal that the best strategy is based on pairing users with highly correlated channels and with the lowest channel gain difference.

The remainder of the paper is organized as follows. Section II characterizes the forward link of the multibeam satellite system under study. The precoding design is tackled in Section III. Next, Section IV conducts the capacity analysis when the receiver applies different decoding strategies. In Section $\mathrm{V}$, scheduling algorithms for overloaded satellite systems are designed. Finally, Section VI presents the numerical results and finally, Section VII draws the conclusion.

\section{SYSTEM MODEL}

Let us consider the forward link of a multibeam satellite system, where the satellite is equipped with an array fed reflector antenna. The number of feeds is denoted by $N$. These feed signals are combined to provide a multiple spot beam coverage of $K$ beams. In this work we have focused on the case where $K=N$.

The multibeam radiation pattern supports data multiplexing, leading to an efficient communication since rate allocation can be performed separately for each signal. In the most general case, $N_{U}$ users are simultaneously served in each beam with different frames. Notice that for $N_{U}>1$, the system is overloaded and the total number of users is higher than the number of feeds. For one-color beam patterns, adjacent beams induce interference, which significantly degrades the system performance. In order to solve this issue, the use of interference mitigation techniques at least at the user terminal (MUD) or at the gateway side (precoding) is mandatory. Similarly to [5], this paper focuses on the combination of both.

Considering that all beams radiate in the same frequency band, the input-output relation at a given time instant can be formulated as

$$
\mathbf{y}=\mathbf{H} \mathbf{x}+\mathbf{n} .
$$

Let $\mathbf{y} \in \mathbb{C}^{K N_{U} \times 1}$ be the vector that stacks column-wise the signals received by all users, $\mathbf{x} \in \mathbb{C}^{N \times 1}$ be the precoded symbol vector and $\mathbf{n} \in \mathbb{C}^{K N_{U} \times 1}$ be the vector that contains the noise terms of each user terminal. The elements of $\mathbf{n}$ are assumed to be Gaussian distributed with zero mean and variance equal to 1 . In addition, noise samples are independent (i.e. $E\left[\mathbf{n n}^{H}\right]=\mathbf{I}_{K N_{U}}$ ) and uncorrelated with the desired signal. The channel matrix can be expressed as follows:

$$
\mathbf{H}=\mathbf{A G},
$$

where $\mathbf{A} \in \mathbb{R}^{K N_{U} \times K N_{U}}$ is a diagonal matrix whose diagonal entries model the atmospheric fading terms. Matrix $\mathbf{G} \in \mathbb{R}^{K N_{U} \times N}$ takes into account the rest of gain and loss factors. Its $(k, n)$-th entry is given by

$$
[\mathbf{G}]_{k, n}=\frac{G_{R} a_{k n}}{4 \pi \frac{d_{k}}{\lambda} \sqrt{K_{B} T_{R} B_{W}}},
$$

for $k=1, \ldots, K N_{U}$ and $n=1, \ldots, N$. As for the rest of the terms, $d_{k}$ accounts for the distance between the $k$-th user terminal and the satellite, $\lambda$ is the carrier wavelength, $K_{B}$ is the Boltzmann constant, $B_{W}$ is the carrier bandwidth, $G_{R}^{2}$ the user terminal receive antenna gain and $T_{R}$ is the receiver noise temperature. The coefficient $a_{k n}$ refers to the gain from the $n$ th feed to the $k$-th user, which depends on the antenna radiation pattern. It is important to mention that the matrix $\mathbf{G}$ has been normalized to the square root of the noise power. For notation convenience, channel matrix $\mathbf{H} \in \mathbb{C}^{K N_{U} \times N}$ can be compactly expressed as

$$
\mathbf{H}=\left[\mathbf{h}_{1}^{T}, \ldots, \mathbf{h}_{K N_{U}}^{T}\right]^{T},
$$

where $\mathbf{h}_{j} \in \mathbb{C}^{1 \times N}$ refers to the channel vector of the $j$-th user. Without loss of generality, we assume that the $i_{1}$-th and the $i_{2}$-th entries of vector $\mathbf{y}$ are associated with users located in the $i$ th spot beam area, for $i_{1}=2(i-1)+1, i_{2}=2(i-1)+2$ and $i=1, \cdots, K$.

In order to minimize inter-beam interference, which is generated by the full frequency reuse scheme and the on-board beamforming generation, precoding is considered. Concerning the notation and the statistical information of the symbols, we denote by $\mathbf{s} \in \mathbb{C}^{K N_{U} \times 1}$ the vector that contains the transmitted symbols, which we assume uncorrelated with unit norm (i.e. $\left.\mathbb{E}\left[\mathbf{s s}^{H}\right]=\mathbf{I}_{K}\right)$. Then, it follows that the precoded symbol vector is given by

$$
\mathbf{x}=\mathbf{W} \mathbf{s}
$$

where $\mathbf{W} \in \mathbb{C}^{N \times K N_{U}}$ is the linear precoding matrix to be designed. For notational convenience, matrix $\mathbf{W}$ can be decomposed as follows:

$$
\mathbf{W}=\left[\mathbf{w}_{1}, \ldots, \mathbf{w}_{K N_{U}}\right] .
$$

Notice that $\mathbf{w}_{i} \in \mathbb{C}^{N \times 1}$ is the precoding vector for the $i$-th user.

\section{PRECODING DESIGN}

Precoding aims at mitigating inter-beam interference, while maintaining the signal to nose ratio (SNR) high. Attending to the performance-complexity trade-off, one of the most suitable precoding schemes is the zero-forcing ( $\mathrm{ZF})$ [4]. The closedform expression of the $\mathrm{ZF}$ can be written as

$$
\mathbf{W}=\gamma\left(\mathbf{H}^{H} \mathbf{H}\right)^{-1} \mathbf{H}^{H},
$$

where $\gamma$ controls the transmit power. Assuming per-feed power constraints, the scaling factor becomes

$$
\gamma^{2}=\frac{P_{\max }}{N \max \left(\operatorname{diag}\left(\left(\mathbf{H}^{H} \mathbf{H}\right)^{-1}\right)\right)} .
$$

Notice that all feeds have the same transmit power constraint, which is $P_{\max } / N$. It has been shown in [5] that precoder designs governed by objective functions different from the $\mathrm{ZF}$ provide higher data rates. However, in this paper priority has been given to the complexity. For this reason, ZF has been favored over other design criteria.

\section{CAPACITY ANALYSIS}

This section is devoted to conduct the capacity analysis of the system described in Section II. It is important to remark that an analogy can be established between the forward link of a multibeam satellite system and the broadcast channel. The broadcast channel capacity can be achieved by means of dirty paper coding. For complexity reasons, this option is discarded 
and instead, we focus on linear precoding designs that can be easily obtained with a closed-form expression. In particular, this section aims at determining the sum-rate in each beam when the system is overloaded, i.e. $N_{U}=2$. Borrowing the notation from Section II, the signals received in the $i$ th beam by the $i_{1}$-th and the $i_{2}$-th users, can be formulated as

$$
\begin{aligned}
& y_{i_{1}}=\mathbf{h}_{i_{1}}\left(\mathbf{w}_{i_{1}} s_{i_{1}}+\mathbf{w}_{i_{2}} s_{i_{2}}\right)+\sum_{l \neq i} \sum_{j=1}^{2} \mathbf{h}_{i_{1}} \mathbf{w}_{l_{j}} s_{l_{j}}+n_{i_{1}}, \\
& y_{i_{2}}=\mathbf{h}_{i_{2}}\left(\mathbf{w}_{i_{2}} s_{i_{2}}+\mathbf{w}_{i_{1}} s_{i_{1}}\right)+\sum_{l \neq i} \sum_{j=1}^{2} \mathbf{h}_{i_{2}} \mathbf{w}_{l_{j}} s_{l_{j}}+n_{i_{2}} .
\end{aligned}
$$

In notation terms, let $s_{l_{j}}$ be the symbol intended for the $l_{j}$ th user and $n_{l_{j}}$ be the additive noise that contaminates the reception of the $l_{j}$-th user. The index $l_{j}$ is associated with the $l$ th user in the $j$ th beam, for $l=1,2$ and $j=1, \cdots, K$. For the ease of exposition, from here onwards we will use $\beta_{j k}^{i}=$ $\left|\mathbf{h}_{i_{j}} \mathbf{w}_{i_{k}}\right|^{2}$ to characterize the channel gains. Since the signal that comes from other beams will not be decoded, the power of the interference-plus-noise term is compactly expressed as

$$
\sigma_{i_{k}}^{2}=1+\sum_{l \neq i} \sum_{j=1}^{2}\left|\mathbf{h}_{i_{k}} \mathbf{w}_{l_{j}}\right|^{2}, \quad k=1,2 .
$$

Building upon (9), the rest of the section is devoted to provide the rate bounds for different decoding strategies, under the Gaussian signaling assumption.

\section{A. Interference as noise}

From the complexity point of view, this is the least complex receiver structure. By treating all the interference terms as noise, the maximum achievable rate becomes

$$
\begin{aligned}
& R_{i_{1}}^{\mathrm{IAN}}=\log _{2}\left(1+\frac{\beta_{11}^{i}}{\beta_{12}^{i}+\sigma_{i_{1}}^{2}}\right), \\
& R_{i_{2}}^{\mathrm{IAN}}=\log _{2}\left(1+\frac{\beta_{22}^{i}}{\beta_{21}^{i}+\sigma_{i_{2}}^{2}}\right) .
\end{aligned}
$$

\section{B. Superposition coding and SIC receiver}

This strategy has been proposed to deal with asymmetric channel gains [6]. To this end, users are classified as strong or weak users, depending on their distance from the base station. In multibeam satellite systems the same approach can be followed, but in this case the channel gains depend on the distance from the center of the beam. To recover the information, the strong user resorts to SIC to cancel the interference from the weak user, while the decoding strategy of the weak user is based on the IAN approach. Therefore, this strategy will be referred to as SIC-IAN. It is important to highlight that the user separation strongly relies on allocating more power to the weak user. Since the number of beams is in the order of hundreds, the complexity required to optimize the power coefficients of all users may not be affordable. Consequently, the power has been equally split among users. With that, the maximum achievable rates that can be jointly achieved are given by

$$
\begin{gathered}
R_{i_{1}}^{\text {SIC-IAN }}=\log _{2}\left(1+\frac{\beta_{11}^{i}}{\sigma_{i_{1}}^{2}}\right) \\
R_{i_{2}}^{\text {SIC-IAN }}=\min \left(R_{i_{2}}^{\text {IAN }}, \log _{2}\left(1+\frac{\beta_{12}^{i}}{\beta_{11}^{i}+\sigma_{i_{1}}^{2}}\right)\right) .
\end{gathered}
$$

Without loss of generality, it has been assumed that the $i_{1}$-th user is closer to the center of the beam than the $i_{2}$-th user. For this reason, the SIC is only implemented by the $i_{1}$-th user.

\section{Simultaneous Non-unique Decoding}

Keeping in mind the input-output relation represented by (9), we compute in this subsection the rate bounds when the simultaneous non-unique decoding (SND) strategy is adopted [7]. The rationale behind SND is that receivers try to jointly decode $s_{i_{1}}$ and $s_{i_{2}}$, but the $i_{1}$-th $\left(i_{2}\right.$-th) user does not care about the errors when decoding $s_{i_{2}}\left(s_{i_{1}}\right)$. A practical scheme to get closer to the boundaries of the capacity region consists in implementing an iterative receiver, where the detector and the decoder exchange soft information [9], [10]. In the last iteration, the information associated with the signal of interest is extracted and the rest is discarded. Concerning the capacity analysis, when the rate assigned to the $i_{2}$-th user is given beforehand, i.e. $R_{i_{2}}^{\mathrm{SND}}$, the maximum achievable rate of the $i_{1}$-th user is given by

$$
R_{i_{1}}^{\mathrm{SND}}= \begin{cases}I_{i_{1} i_{2}} & R_{i_{2}}^{\mathrm{SND}} \leq \log _{2}\left(1+\frac{\beta_{12}^{i}}{\sigma_{i_{1}}^{2}}\right), \\ R_{i_{1}}^{\mathrm{IAN}} & R_{i_{2}}^{\mathrm{SND}}>\log _{2}\left(1+\frac{\beta_{12}^{i}}{\sigma_{i_{1}}^{2}}\right)\end{cases}
$$

where

$$
\begin{gathered}
I_{i_{1} i_{2}}=\min \left\{\log _{2}\left(1+\frac{\beta_{11}^{i}}{\sigma_{i_{1}}^{2}}\right), R_{i_{1}}^{\mathrm{MAC}}-R_{i_{2}}^{\mathrm{SND}}\right\}, \\
R_{i_{1}}^{\mathrm{MAC}}=\log _{2}\left(1+\frac{\beta_{11}^{i}+\beta_{12}^{i}}{\sigma_{i_{1}}^{2}}\right) .
\end{gathered}
$$

From (13) it can be inferred that joint detection is performed if $s_{i_{2}}$ can be reliably decoded. Otherwise, $s_{i_{2}}$ is treated as noise. Analogously to (13), given the rate of the $i_{1}$-th user, i.e. $R_{i_{1}}^{\mathrm{SND}}$, then $R_{i_{2}}^{\mathrm{SND}}$ is expressed as follows:

$$
R_{i_{2}}^{\mathrm{SND}}= \begin{cases}I_{i_{2} i_{1}} & R_{i_{1}}^{\mathrm{SND}} \leq \log _{2}\left(1+\frac{\beta_{21}^{i}}{\sigma_{i_{2}}^{2}}\right) \\ R_{i_{2}}^{\mathrm{IAN}} & R_{i_{1}}^{\mathrm{SND}}>\log _{2}\left(1+\frac{\beta_{21}^{i}}{\sigma_{i_{2}}^{2}}\right),\end{cases}
$$

Notice that the closed-form expression of $I_{i_{2} i_{1}}$ and $R_{i_{2}}^{\mathrm{MAC}}$ can be easily deduced from (14) and (15). Hence, due to space limitations, we refrain from including the corresponding definitions.

It is particularly noteworthy that the rate region that is jointly achievable by $i_{1}$-th and $i_{2}$-th users is the intersection of (13) and (16). The computation of the jointly achievable rates allows us to select the best rate tuple $\left(R_{i_{1}}^{\mathrm{SND}}, R_{i_{2}}^{\mathrm{SND}}\right)$. In this regard, Fig. 1 shows how the capacity region may look like depending on the magnitude of the interfering signal. That is, Fig. 1.a corresponds to the case where intra-beam interference is not negligible. By contrast, the capacity region depicted 


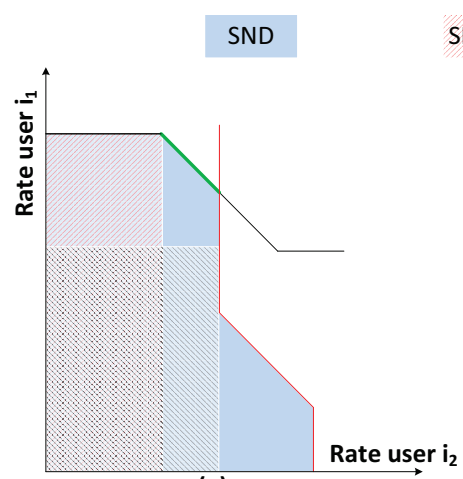

(a)

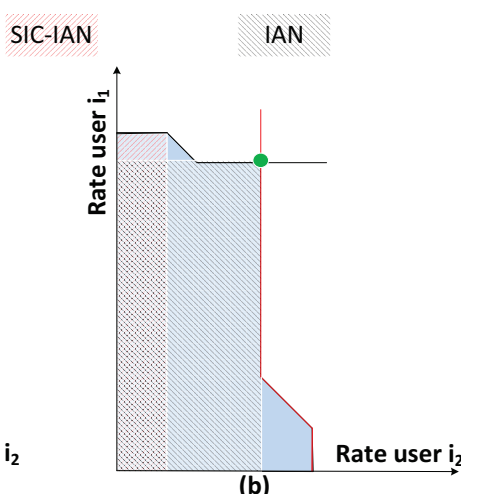

(b)

Fig. 1. Capacity region. a) High intra-beam interference. b) Low intra-beam interference.

in Fig. 1.b determines the achievable rates when the impact of intra-beam interference is very low, i.e. $\beta_{11}^{i}>>\beta_{12}^{i}$ and $\beta_{22}^{i}>>\beta_{21}^{i}$. Interestingly, the SIC-IAN approach can attain the highest sum-rate in Fig. 1.a, which is highlighted in green. However, when users almost do not interfere each other, as in Fig. 1.b, the best strategy is to opt for receivers that adopt the IAN strategy. It is worth emphasizing that any rate achieved by SIC-IAN and IAN strategies can be achieved by SND.

\section{Scheduling in Overloaded SATEllite Systems}

Bearing in mind Section IV, it is difficult to establish an a priori heuristic to determine which users should be active to maximize the sum-rate. This differs from the case where receivers perform SUD and the number of users that are simultaneously served in each beam is $N_{U}=1$. In this scenario, ZF precoding jointly with nearly orthogonal user grouping asymptotically approaches the capacity of dirty paper coding [11]. An additional challenge that the scheduling has to face in NOMA multibeam satellite systems is the elevated number of beams in which the coverage area is divided. This prevents the scheduling from using brute-force search.

In this context, our first proposal is described in Algorithm 1 , which is referred to as minimum Euclidean norm (MEN) method. Following this approach, in each beam we randomly select one user and, next, we find the user in the same beam that presents the lowest Euclidean distance. The reasoning behind this approach is that the precoder should be able to mitigate inter-beam interference more effectively, whenever the users paired with the same beam have similar channel vectors. In Algorithm 1 we denote by $\mathcal{M}_{k}$ the set of users to be served at the $k$-th beam and by $\mathcal{S}_{k}$ the selected users.

An alternative to the metric used in Algorithm 1 is based on considering the channel vector collinearity and the channel gain separately. Indeed, considering that

$$
\left\|\mathbf{h}_{u_{k}}-\mathbf{h}_{v_{k}}\right\|^{2}=\left\|\mathbf{h}_{u_{k}}\right\|^{2}+\left\|\mathbf{h}_{u_{l}}\right\|^{2}-2 \operatorname{Re}\left\{\mathbf{h}_{u_{k}} \mathbf{h}_{u_{l}}^{H}\right\},
$$

we can establish that given a collinearity factor $\operatorname{Re}\left\{\mathbf{h}_{u_{k}}^{H} \mathbf{h}_{u_{l}}\right\}$, the MEN method gives priority to users with low channel gains. As a matter of fact, it is not clear whether the decoding strategies will benefit from a large or a low power imbalance. Because of this, the Algorithm 2 describes minimum power imbalance (MinPI) and maximum power imbalance (MaxPI)

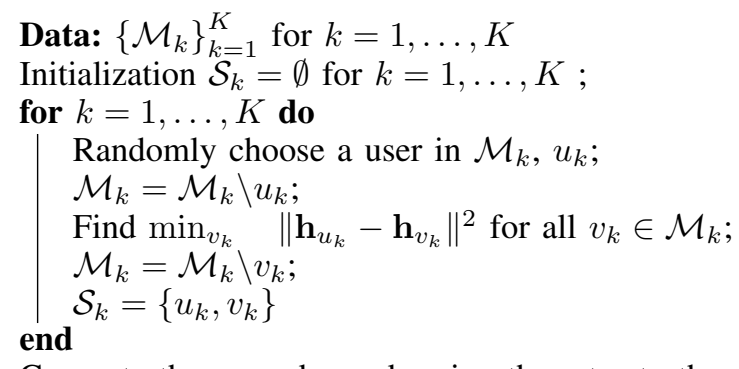

Compute the precoder and assign the rates to the users;

Algorithm 1: Minimum Euclidean norm method.

approaches. Similarly to MEN, we first randomly choose one user $u_{k}$. However, for MaxPI and MinPI we perform an initial search to find the channels that have an scalar product $\left|\mathbf{h}_{u_{k}} \mathbf{h}_{u_{l}}^{H}\right| /\left\|\mathbf{h}_{u_{k}}\right\|\left\|\mathbf{h}_{u_{l}}\right\|$ larger than a parameter $\nu$. In this paper we have considered $\nu=0.8$. The aim of this condition is to identify highly correlated channels, so that the precoder can mitigate the detrimental effects induced by interbeam interference. Thanks to MUD, receivers can deal with intra-beam inerference. Among the selected users, which are gathered in $\mathcal{B}_{k}$, we select the one with lowest (highest) channel gain imbalance for the MinPI (MaxPI).

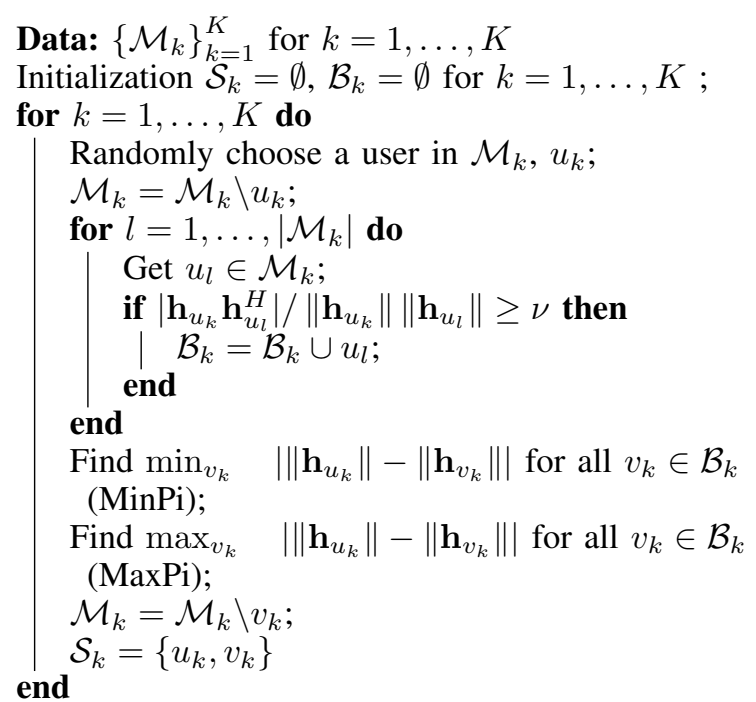

Compute the precoder and assign the rates to the users; Algorithm 2: Minimum and maximum power imbalance method.

\section{NUMERICAL RESULTS}

This section presents the numerical results considering the decoding strategies and the scheduling algorithms described in Sections IV and V, respectively. To evaluate the aforementioned techniques, a Ka-band real coverage area provided by a geostationary satellite is considered. The data has been obtained in a study performed by the European space agency (ESA). The parameters are the same than those used in [5]. We assume that at each time instant a total bandwidth of 500 $\mathrm{MHz}$ is shared by all beams. The coverage area consists of $K=245$ beams with $N=245$ feeds. We assume that $N_{U}=2$ users are simultaneously served in each beam and we perform a Monte Carlo simulation with 1000 runs. For every run, we 


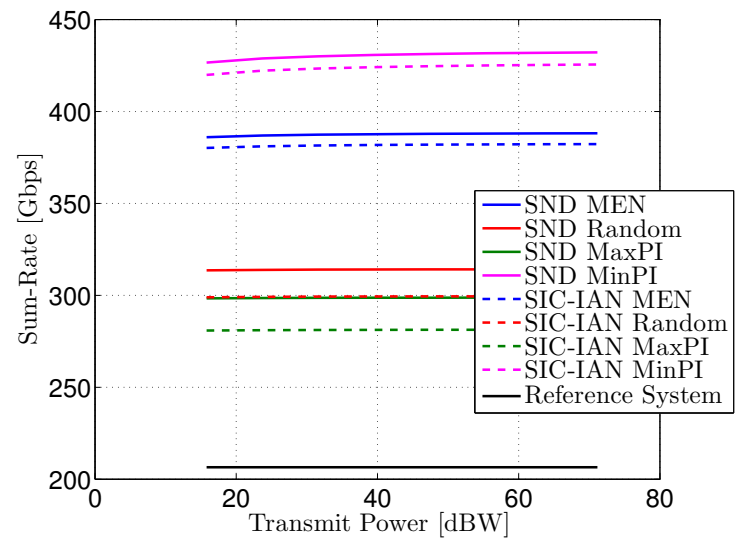

Fig. 2. Sum-rate versus transmit power.

consider that the scheduler has a total number of $\left|\mathcal{M}_{k}\right|=50$ users to be served, for $k=1, \ldots, K$.

Fig. 2 depicts the sum-rate for different scheduling and decoding strategies. Notice that the per feed available power ranges from 18 to $72 \mathrm{dBWatts}$. The results show that the scenario is limited by the interference. Given the scheduler, it can be observed that the SND strategy always yields higher data rates than SIC-IAN, which is in line with Fig. 1. We can observe in Fig. 2 that the scheduling that leads to the highest data rates is MinPI. As reported in [8], it might be convenient to pair users with the largest channel gain difference. However, the numerical results reveal that this is not the case for the scenario under study. From the authors point of view this is due to the absence of power control, which penalizes the rate of the weak user.

For comparison purposes, we include the random scheduling (i.e. a pair of users are randomly chosen within each beam). As a benchmark, we include the reference system where $N_{U}=1$. In this case, the rate is divided by two to highlight that two time slots would be required to support $K N_{U}$ users with $N$ feeds. As a result, the rate is significantly reduced with respect to the proposed overloaded satellite system.

Finally, Fig. 3 presents the user data rate variance over the coverage area, when the transmit power is set to $72 \mathrm{dBWatts}$. This metric gives an idea of the fairness. It can be observed that the SND strategy exhibits the lowest variance, regardless of the scheduling algorithm. Therefore, SND achieves a better balance between the highest and the lowest achievable rates, when compared to the SIC-IAN strategy.

\section{CONCLUSIONS}

This paper evaluates different scheduling algorithms and decoding strategies for the forward link of multibeam satellite systems. When all the beams share the same frequency and the number of users that are simultaneously served is higher than the number of feeds, (i.e. $K N_{U}>N$ ), the precoder is not able to eliminate the interference. To overcome this issue, residual inter-beam interference is treated as noise, and receivers are able to perform MUD techniques to deal with intra-beam interference. In this case, scheduling algorithms conceived for SUD techniques cannot be applied. In this sense,

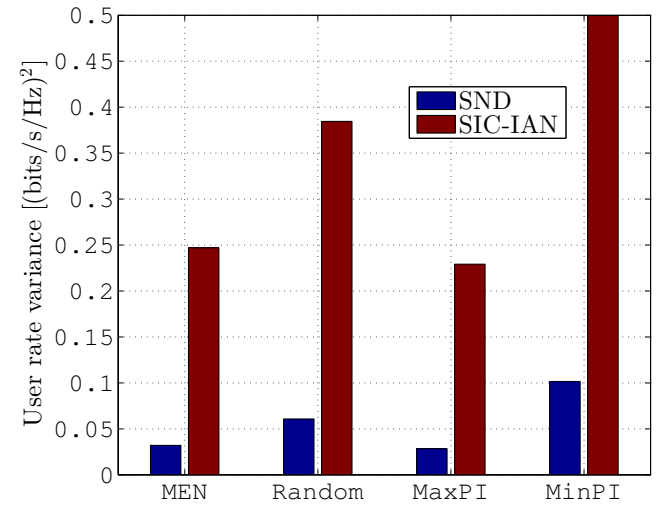

Fig. 3. User rate variance with different scheduling algorithms.

new algorithms to pair users with beams have been proposed. Numerical results reveal that SND outperforms SIC-IAN in terms of rate and fairness. In addition, when the system is overloaded, the best strategy is to pair users with highly correlated channels and the lowest channel gain difference.

\section{REFERENCES}

[1] G. Colavolpe, A. Modenini, A. Piemontese, and A. Ugolini, "Multiuser detection in multibeam satellite systems: Theoretical analysis and practical schemes," in IEEE 16th International Workshop on Signal Processing Advances in Wireless Communications (SPAWC), June 2015, pp. 525-529.

[2] M. Caus, A. I. Perez-Neira, M. Angelone, and A. Ginesi, "An innovative interference mitigation approach for high throughput satellite systems," in IEEE 16th International Workshop on Signal Processing Advances in Wireless Communications (SPAWC), June 2015, pp. 515-519.

[3] G. Colavolpe, A. Modenini, A. Piemontese, and A. Ugolini, "On the application of multiuser detection in multibeam satellite systems," in IEEE International Conference on Communications (ICC), June 2015, pp. 898-902.

[4] B. Devillers, A. Perez-Neira, and C. Mosquera, "Joint Linear Precoding and Beamforming for the Forward Link of Multi-Beam Broadband Satellite Systems," in IEEE Global Telecommunications Conference (GLOBECOM 2011), Dec 2011.

[5] M. A. Vázquez, M. Caus, and A. Pérez-Neira, "Performance Analysis of Joint Precoding and MUD Techniques in Multibeam Satellite Systems," in IEEE Global Telecommunications Conference (GLOBECOM 2016), Dec 2016.

[6] Y. Saito, Y. Kishiyama, A. Benjebbour, T. Nakamura, A. Li, and K. Higuchi, "Non-orthogonal multiple access (NOMA) for cellular future radio access," in IEEE 77th Vehicular Technology Conference (VTC Spring), June 2013, pp. 1-5.

[7] W. Nam, D. Bai, J. Lee, and I. Kang, "Advanced interference management for 5G cellular networks," IEEE Communications Magazine, vol. 52, no. 5, pp. 52-60, May 2014.

[8] B. Kim, S. Lim, H. Kim, S. Suh, J. Kwun, S. Choi, C. Lee, S. Lee, and D. Hong, "Non-orthogonal multiple access in a downlink multiuser beamforming system," in IEEE Military Communications Conference. IEEE, 2013, pp. 1278-1283.

[9] S. ten Brink, J. Speidel, and R.-H. Yan, "Iterative demapping and decoding for multilevel modulation," in IEEE GLOBECOM 1998, vol. 1, 1998, pp. 579-584 vol.1.

[10] X. Wang and H. V. Poor, "Iterative (turbo) soft interference cancellation and decoding for coded CDMA," IEEE Transactions on Communications, vol. 47, no. 7, pp. 1046-1061, Jul 1999.

[11] T. Yoo and A. Goldsmith, "On the optimality of multiantenna broadcast scheduling using zero-forcing beamforming," IEEE Journal on Selected Areas in Communications, vol. 24, no. 3, pp. 528-541, March 2006. 